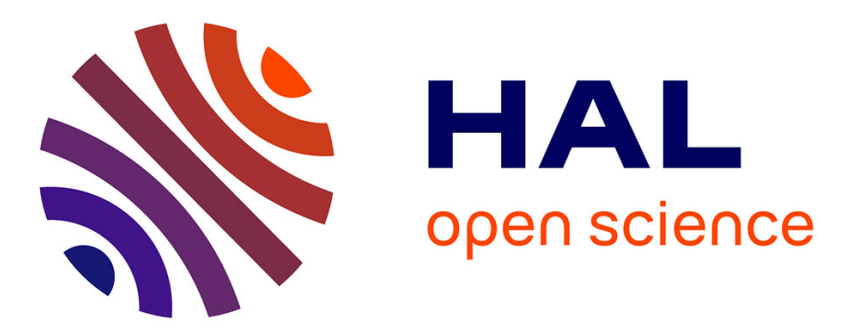

\title{
Probing Electro-Magnetic Local Density of Optical States with Mixed ED-MD Emitters
}

Dongfang Li, Sinan Karaveli, Sébastien Cueff, Wenhao Li, Rashid Zia

\section{To cite this version:}

Dongfang Li, Sinan Karaveli, Sébastien Cueff, Wenhao Li, Rashid Zia. Probing Electro-Magnetic Local Density of Optical States with Mixed ED-MD Emitters. CLEO: QELS_Fundamental Science, 2019, San Jose, United States. pp.FW3C.4, 10.1364/CLEO_QELS.2019.FW3C.4 . hal-02408499

\section{HAL Id: hal-02408499 \\ https://hal.science/hal-02408499}

Submitted on 18 Nov 2020

HAL is a multi-disciplinary open access archive for the deposit and dissemination of scientific research documents, whether they are published or not. The documents may come from teaching and research institutions in France or abroad, or from public or private research centers.
L'archive ouverte pluridisciplinaire HAL, est destinée au dépôt et à la diffusion de documents scientifiques de niveau recherche, publiés ou non, émanant des établissements d'enseignement et de recherche français ou étrangers, des laboratoires publics ou privés. 


\title{
Probing Electro-Magnetic Local Density of Optical States with Mixed ED-MD Emitters
}

\author{
Dongfang Li, ${ }^{1,2}$ Sinan Karaveli, ${ }^{1}$ Sébastien Cueff ${ }^{1,3}$ Wenhao Li, ${ }^{1}$ and Rashid Zia ${ }^{1}$ \\ ${ }^{1}$ School of Engineering and Department of Physics, Brown University, Providence, RI 02912, USA \\ ${ }^{2}$ Center for Integrated Nanotechnologies, Los Alamos National Laboratory, Los Alamos, NM 87545, USA \\ ${ }^{3}$ Institut des Nanotechnologies de Lyon, Ecole Centrale de Lyon, 69134 Ecully, France \\ dongfangli@lanl.gov
}

\begin{abstract}
We experimentally demonstrated that the lifetime of quantum emitters with strongly mixed electric dipole (ED) and magnetic dipole (MD) transitions can directly probe the combined electro-magnetic local density of optical states.

OCIS codes: (250.0250) Optoelectronics; (260.2510) Fluorescence; (260.3160) Interference.
\end{abstract}

Purcell effect shows the spontaneous decay rate of a quantum emitter depends on its local density of optical states (LDOS), which indicates that the spontaneous decay rate can be selectively enhanced or suppressed by properly engineering its local optical environment. As demonstrated in Drexhage's pioneering experiments, the electric LDOS oscillates as a function of the separation distance between the emitter and metal-dielectric interface (red line in Fig. 1(a)), thus leading to the measurable oscillations of corresponding lifetimes (red line in Fig. 1(b)). The lifetime oscillations refer to the enhancement and suppression of spontaneous decay rate of light emitters at varied locations. In the literature, the fitting to the oscillations has been employed to infer quantum efficiency of light emitters. However, these oscillations are not a fundamental nature of electromagnetic mode density. The measured electric LDOS using the common purely ED or ED-dominant emitters is only the projection of the combined electro-magnetic LDOS onto an isotropic electric dipole (Fig. 1(a)).

In the past decade, the magnetic light matter interaction has drawn great attention thanks to the advance of metamaterials. In addition to those magnetic resonators (e.g., split-ring resonators and parallel nanorods), strong MD transitions exist as well in transition-metal and lanthanide ions [1]. In order to probe the combined electro-magnetic LDOS, strongly mixed ED-MD emitters are required to access to electric and magnetic LDOS simultaneously.

Here we experimentally investigated the lifetimes of two strongly mixed ED-MD emitters: divalent nickel ions in magnesium oxide $\left(\mathrm{Ni}^{2+}: \mathrm{MgO}\right)$ and trivalent erbium ions in yttrium oxide $\left(\mathrm{Er}^{3+}: \mathrm{Y}_{2} \mathrm{O}_{3}\right)$ [2]. Staircaselike samples with 44 distinct regions (i.e., with varied spacer thicknesses $d$ and/or top interfaces) were fabricated for the two quantum emitters, respectively. Time-dependent photoluminence was then measured for all distinct areas of the samples to extract the corresponding lifetimes. The measured lifetime of the mixed ED-MD emitter does not solely depend on electric or magnetic LDOS, but rather follows the combined electromagnetic LDOS. Almost no lifetime oscillations was observed in the measured lifetimes of ${ }^{3} \mathrm{~T}_{2} \rightarrow{ }^{3} \mathrm{~A}_{2}$ transitions in $\mathrm{Ni}^{2+}: \mathrm{MgO}$, as shown in Fig. 1(c) (black dots).

This is further confirmed by a technologically and scientifically important emitter $\operatorname{Er}^{3+}: \mathrm{Y}_{2} \mathrm{O}_{3}$. Of great interest, the complex electronic levels of $\mathrm{Er}^{3+}$ make it support both purely ED transitions $\left({ }^{4} \mathrm{~S}_{3 / 2} \rightarrow{ }^{4} \mathrm{I}_{15 / 2}\right)$ at visible regime and strongly mixed ED-MD transitions $\left({ }^{4} \mathrm{I}_{13 / 2} \rightarrow{ }^{4} \mathrm{I}_{15 / 2}\right)$ at telecom wavelengths [3]. The measured lifetime of strongly mixed ED-MD transitions ${ }^{4} \mathrm{I}_{13 / 2} \rightarrow{ }^{4} \mathrm{I}_{15 / 2}$ in $\mathrm{Er}^{3+}: \mathrm{Y}_{2} \mathrm{O}_{3}$ at telecom wavelengths follows the combined electromagnetic LDOS. In contrast, the measured lifetime of the purely ED transitions at visible regime $\left({ }^{4} \mathrm{~S}_{3 / 2} \rightarrow{ }^{4} \mathrm{I}_{15 / 2}\right)$ originating from the same $\mathrm{Er}^{3+}$ emitters solely depends on the electric LDOS. The extracted MD contributions from the theoretical fitting of measured lifetimes match the results obtained by the recently developed energy-momentum spectroscopy $[3,4]$.

The findings here show that, for emitters with strongly mixed ED-MD transitions as well as characterized quantum efficiency and bulk lifetime, a single lifetime measurement can probe the combined electro-magnetic LDOS at the point of interest in a complex optical system, such as metamaterials, epsilon-near-zero materials, and topological photonics. 

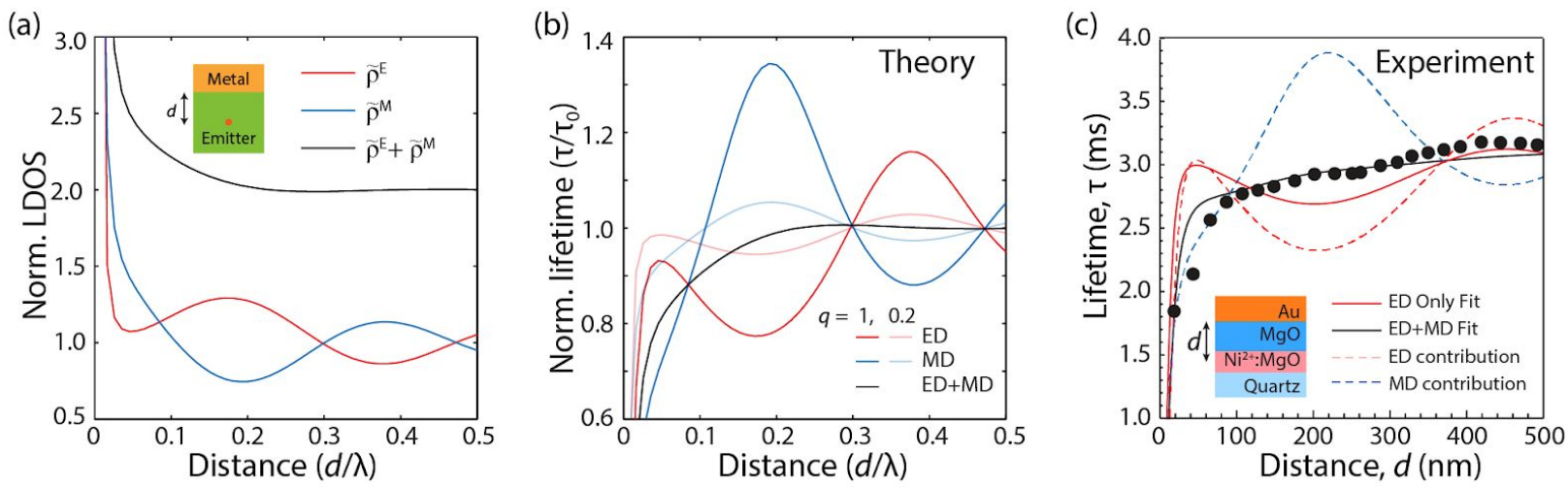

Fig. 1 (a) Normalized electric (red) and magnetic (blue) LDOS, together with their summation (black). Inset is the sample schematic. (b) Normalized lifetimes for purely ED (red), MD (blue) and strongly mixed ED-MD (black) emitters with varied quantum efficiencies q $=1$ and 0.2 . In the simulation of LDOS and lifetimes, the dielectric constants for the emitter's host and metallic mirror are $\varepsilon=2.25$ and $-97+11.5 \mathrm{i}$ respectively. (c) Measured lifetimes (black dots) for mixed ED-MD emitter $\mathrm{Ni}^{2+}: \mathrm{MgO}$ at near infrared wavelengths. The theoretical fits for ED-MD mixed model and purely ED model are shown by black and red lines, respectively. The corresponding ED and MD contributions in the ED-MD mixed model are shown by the red and blue dashed lines. Note that the absence of the lifetime oscillation can not be attributed to the low quantum efficiency due to the relatively long measured lifetime. Figure reproduced from Ref. [2]

\section{References}

[1] C. M. Dodson and R. Zia, "Magnetic dipole and electric quadrupole transitions in the trivalent lanthanide series: Calculated emission rates and oscillator strengths," Phys. Rev. B 86(12), 125102 (2012).

[2] D. Li, S. Karaveli, S. Cueff, W. Li, and R. Zia, "Probing the combined electromagnetic local density of optical states with quantum emitters supporting strong electric and magnetic transitions," Phys. Rev. Lett. 121(22), 227403 (2018).

[3] D. Li, M. Jiang, S. Cueff, C. M. Dodson, S. Karaveli, and R. Zia, "Quantifying and controlling the magnetic dipole contribution to 1.5- $\mu \mathrm{m}$ light emission in erbium-doped yttrium oxide," Phys. Rev. B 89(16), 161409(R) (2014).

[4] T. H. Taminiau, S. Karaveli, N. F. van Hulst, and R. Zia, "Quantifying the magnetic nature of light emission," Nature Commun. 3, 979 (2012). 